\title{
An Efficient Game Form for Unicast Service Provisioning
}

\author{
Ali Kakhbod and Demosthenis Teneketzis \\ Department of Electrical Engineering and Computer Science, \\ University of Michigan, Ann Arbor, MI, USA. \\ Email: $\{$ akakhbod, teneket\}aumich.edu
}

\begin{abstract}
We consider the decentralized bandwidth/rate allocation problem in unicast service provisioning with strategic users. We present a mechanism/game form which possesses the following properties when the users' utilities are concave: (1) It implements in Nash equilibria the solution of the corresponding centralized rate allocation problem in unicast service provisioning. (2) It is individually rational. (3) It is budget-balanced at all Nash equilibria of the game induced by the mechanism/game form as well as off equilibrium. When the users' utilities are quasi-concave the mechanism possesses properties (2) and (3) stated above. Moreover, every Nash equilibrium of the game induced by the proposed mechanism results in a Walrasian equilibrium.
\end{abstract}

\section{INTRODUCTION}

\section{A. Motivation and Challenges}

Most of today's networks, called integrated services networks support the delivery of a variety of services to their users each with its own quality of service (QoS) requirements (e.g. delay, percentage of packet loss, jitter, etc). As the number of services offered by the network and the demand for the services increase, the need for efficient network operation increases. One of the key factors that contributes to efficient network operation is the efficient utilization of the network's resources. 
The design of resource allocation mechanisms which guarantee the delivery of different services, each with its own QoS requirements, and maximize some network-wide performance criterion (e.g. the network's utility to its users) is an important and challenging task. The challenges come from: (1) the fact that the network is an informationally decentralized system; (2) the network's users may behave strategically (i.e. they may behave selfishly). Networks are informationally decentralized systems. Each user's utility is its own private information. Users are unaware of each others' utilities and of the resources (e.g. bandwidth, buffers, spectrum) available to the network. The network (network manager) knows the network's topology and its resources but is unaware of the users' utilities. If information were centralized, the resource allocation problem could be formulated and solved as a mathematical programming problem or as a dynamic programming problem. Since information is not centralized such formulations are not possible. The challenge is: (1) to determine a message exchange process among the network and users and an allocation rule (based on the outcome of the message exchange process) that eventually lead to a resource allocation that is optimal for the centralized problem, (2) To take into account, in the determination of the allocation mechanism, the possible strategic (selfish) behavior of the network's users.

The topic of resource allocation for informationally decentralized systems has been explored in great detail by mathematical economists in the context of mechanism design. Decentralized resource allocation problems arising in networks have recently attracted significant attention among engineers. Below we present a brief survey of the existing literature on decentralized network resource allocation and briefly refer to texts and survey articles written by mathematical economists on mechanism design. A more detailed discussion of the existing literature on network resource allocation, and a comparison of the results of our paper with this literature will be presented in section $\mathrm{I}-\mathrm{C}$ of the paper.

Within the context of communication networks most of existing literature (e.g. [1]-[20] and [21]) has approached the design of decentralized resource allocation mechanisms under the assumption that the network (network manager) and its users are cooperative (non-strategic), that is, they obey the rules of the proposed decentralized resource allocation mechanism.

Decentralized resource allocation problems with strategic users are solved through the development of game forms/mechanisms which together with the users' utilities give rise to games. Depending on the information available to the users, the game induced by the mechanism is either 
one of complete information or incomplete information ( [22]); the nature of the game dictates the behavioral/equilibrium concept (e.g. Nash equilibrium (NE), subgame perfect equilibrium, Bayesian Nash equilibrium, sequential equilibrium) that is appropriate/suitable for the solution of the resource allocation problem. The allocation mechanisms specify; (1) the allocations made at all equilibrium and non-equilibrium points of the game; and (2) the tax (positive or negative) each user pays as a result of his/her participation in the game. Desirable properties of resource allocation mechanisms with strategic users are: (1) the allocations corresponding to all equilibria of the resulting game must be globally optimal; (2) the users must voluntarily participate in the allocation process; (3) the budget must be balanced, that is the sum of taxes paid by the users must be zero (there should be no money left to be thrown away at the end of the allocation process).

Recently, within the context of communication networks, researchers have investigated decentralized resource allocation problems under the assumption that users behave strategically (i.e. they are not price-takers, they do not necessarily obey the rules of the mechanism but have to be induced to follow the rules). Within the context of wired networks, decentralized resource allocation mechanisms have been proposed and analyzed in [6], [8], [23]-[30] and [31]. A more detailed discussion of these references and comparison with the results of this paper will be presented in section [-C. Decentralized power allocation mechanisms for wireless networks with strategic users where every user's transmission creates interference to every other user or to a subset of the network's users were proposed and analyzed in [18], [32].

Resource allocation problems in informationally decentralized systems has been explored in great detail by mathematical economists in the context of mechanism design. There is an enormous economics literature for the situation where users are cooperative (e.g [33], [34] and references therein) as well as the situation where users are strategic (e.g [33] $-[35]$, and references therein). For a survey on the relationship of mechanism design and decentralized resource allocation in communication networks we refer the reader to [36].

\section{B. Contribution of the paper}

We investigate the unicast service provisioning problem in wired networks with arbitrary topology and strategic users. The main contribution of this paper is the discovery of a decentralized rate allocation mechanism for unicast service provisioning in networks with arbitrary/general 
topology and strategic users, which possesses the following properties.

When each user's utility is concave, then:

- (P1) The mechanism implements the solution of the centralized unicast service provisioning problem in Nash equilibria. That is, the allocation corresponding to each NE of the game induced by the mechanism is a globally optimal solution of the corresponding centralized resource allocation problem.

- (P2) The mechanism is individually rational, that is, the network users voluntarily participate in the rate allocation process.

- (P3) The mechanism is budget-balanced ${ }^{1}$ at all feasible allocations, that is, at all the allocations that correspond to NE messages/strategies as well as at all the allocations that correspond to off-equilibrium messages/strategies.

When each user's utility is quasi-concave but differentiable, then:

- The mechanism possesses properties (P2) and (P3).

- (P4) Every NE of the game induced by the mechanism results in a Walrasian equilibrium ( [12] Ch. 15), consequently, a Pareto optimal allocation.

To the best of our knowledge, none of the decentralized resource allocation mechanisms proposed so far for the unicast service provisioning problem in communication networks possesses simultaneously all three properties (P1)-(P3) when the network's topology is general/arbitrary, the users are strategic and their utilities are concave. Furthermore, we are not aware of the existence of any publications in unicast service provisioning containing the analysis of a decentralized rate allocation mechanism when the users are strategic and their utilities are quasi-concave.

We now compare in more detail our contributions with the existing literature.

\section{Comparison with related work}

We now explain why the proposed mechanism and the above results are distinctly different from all game forms/mechanisms proposed so far for the unicast service provisioning problem with strategic users.

Most of the previous work on the unicast service provisioning problem in networks with general topology is based on Vickrey-Clark-Groves(VCG)-type mechanisms, [25]-[27], [31],

\footnotetext{
${ }^{1}$ In a budget-balanced mechanism the sum of the taxes paid by a subset of users is equal to the sum of subsidies received by the rest of the users.
} 
[37]-[41]. The game forms/mechanisms proposed in [31] and [25] induce games that establish the existence of a unique Nash equilibrium at which the allocation is globally optimal under some conditions; but these mechanisms are not budget-balanced even at equilibrium. The mechanisms/game forms proposed in [26], [27], [37], [38] induce games that have multiple NE; these mechanisms are not budget-balanced even at equilibrium, and the allocations corresponding to the Nash equilibria are not always globally optimal ( that is these mechanisms do not implement in Nash equilibria the solution of the centralized unicast service provisioning problem). Our mechanism is not of the VCG-type, thus, it is philosophically different from those of [25]-[27], [31], [37], [38].

The work in [28], [29] and [42] deals with single link networks. For these single-link networks the authors of [29] proposed a class of efficient (optimal) allocation mechanisms, called ESPA, for the allocation of a single divisible good. ESPA mechanisms were further developed in [28]. It is not currently known whether ESPA mechanisms implement in Nash equilibria the optimal solution of the unicast service provisioning problem in networks with arbitrary/general topology. The network model considered in this paper has arbitrary/general topology.

In [23], [24] the authors show that when the resource allocation mechanism proposed in [6] is considered under the assumption that the users are strategic and NE is the equilibrium concept, the allocations corresponding to any NE are different from any allocations that are optimal solutions of the corresponding centralized unicast service provisioning problem; that is, the allocation corresponding to any NE suffer from a certain efficiency loss. Particularly, in [24] it is shown that there exists a lower bound on the efficiency loss. The mechanism we propose in this paper is distinctly different from those of [23], [24]. Our mechanism results in the same performance as optimal centralized allocations, that is, the allocations corresponding to any NE of the game induced by our mechanism are efficient.

Philosophically, our work is most closely related to [30], but it is distinctly different from [30] for the following reasons: (1) the game form proposed in our paper is distinctly different from that of [30]. (2) The mechanism of [30] is not balanced off equilibrium. (3) In the mechanism of [30] there is no coupling among the games that are being played at different links. In our mechanism such a coupling exists (see section $[\mathrm{III}$ ), and results in a balanced-budget off equilibrium.

Finally, we are not aware of any publication, other than this paper, containing the analysis of a decentralized rate allocation mechanism for unicast service provisioning when the users are 
strategic and their utilities are quasi-concave.

\section{Organization of the paper}

The rest of the paper is organized as follows. In section II we formulate the unicast service provisioning problem with strategic users. In section III we describe the allocation mechanism/game form we propose for the solution of the unicast service provisioning problem. In section IV] we analyze the properties of the proposed mechanism. In section $\mathrm{V}$ we discuss how the game form/mechanism presented in this paper can be implemented in a network. In section VI we investigate the properties of the game form proposed in this paper when the users' utilities $\mathbf{U}_{i}, i \in \mathcal{N}$, are quasi-concave. We conclude in section $\mathrm{VII}$.

\section{ThE UNICAST PROBLEM WITH STRATEGIC NETWORK USERS, PROBLEM FORMULATION}

In this section we present the formulation of the unicast problem in wired communication networks with strategic users. We proceed as follows, In section II-A we formulate the centralized unicast service provisioning problem the solution of which we want to implement in Nash equilibria. In section $\Pi \mathrm{I}-\mathrm{B}$ we formulate the decentralized unicast service provisioning problem with strategic network users, we state our assumptions, our objective and provide an interpretation of the equilibrium concept (Nash equilibrium) in which we want to implement the solution of the centralized problem of section II-A.

\section{A. The centralized problem}

We consider a wired network with $N, N>3$, users. The set of these users is denoted by $\mathcal{N}$, i.e. $\mathcal{N}=\{1,2, \cdots, N\}$. The network topology, the capacity of the network links, and the routes assigned to users' services are fixed and given. The users' utility functions have the form

$$
\mathcal{V}_{i}\left(x_{i}, t_{i}\right)=\mathbf{U}_{i}\left(x_{i}\right)-t_{i}, \quad i=1,2, \cdots, N
$$

The term $\mathbf{U}_{i}\left(x_{i}\right)$ expresses user $i$ 's satisfaction from the service $x_{i}$ it receives. The term $t_{i}$ represents the $\operatorname{tax}$ (money) user $i$ pays for the services it receives. We assume that $\mathbf{U}_{i}$ is a concave and increasing function of the service $x_{i}$ user $i$ receives, and $t_{i} \in \mathbb{R}$. When $t_{i}>0$ user $i$ pays money for the services it receives; this money is paid to other network users. When $t_{i}<0$ user $i$ receives money from other users. Overall, the amount of money paid by some of 
the network users must be equal to the amount of money received by the rest of the users so that $\sum_{i \in \mathcal{N}} t_{i}=0$. Denote by $\mathbf{L}$ the set of links of the network, by $\mathbf{c}^{l}$ the capacity of link $l$, and by $\mathcal{R}_{i}$ the set of links $l, l \in \mathbf{L}$, that form the route of user $i, i=1,2, \cdots, N$ (as pointed out above each user's route is fixed). We assume that a central authority (the network manager) has access to all of the above information. The objective of this authority is to solve the following centralized optimization problem that we call Max.

$$
\begin{array}{ll}
\operatorname{Max} \max _{x_{i}} & \sum_{i=1}^{N} \mathbf{U}_{i}\left(x_{i}\right) \\
\text { subject to } & \\
& \sum_{i: l \in \mathcal{R}_{i}} x_{i} \leq \mathbf{c}^{l}, \quad \forall l \in \mathbf{L}, \\
& x_{i} \geq 0, \quad \forall i \in \mathcal{N}, \\
& \sum_{i=1}^{N} t_{i}=0, t_{i} \in \mathbb{R}, \quad \forall i \in \mathcal{N} .
\end{array}
$$

The inequalities in (3) express the capacity constraints that must be satisfied at each network link. The inequalities in (4) express the fact that the users' received services $x_{i}, i \in \mathcal{N}$ must be nonnegative. The equality in (5) express the fact that the budget must be balanced, i.e. the total amount of money paid by some of the users must be equal to the amount of money received by the rest of the users.

Let $\mathcal{U}$ denote the set of functions

$$
\mathbf{U}: \mathbb{R}_{+} \cup\{0\} \rightarrow \mathbb{R}_{+} \cup\{0\}
$$

where $\mathbf{U}$ is concave and increasing. Let $\mathbf{T}$ denote the set of all possible network topologies, network resources and user routes. Consider problem Max for all possible realizations

$$
\left(\mathbf{U}_{1}, \cdots, \mathbf{U}_{N}, T\right) \in \mathcal{U}^{N} \times \mathbf{T}
$$

of the users' utilities, the network topology, its resources and the users' routes. Then, the solution of Max for each $\left(\mathbf{U}_{1}, \mathbf{U}_{2} \cdots, \mathbf{U}_{N}, T\right) \in \mathcal{U}^{N} \times \mathbf{T}$ defines a map

$$
\pi: \mathcal{U}^{N} \times \mathbf{T} \rightarrow \mathcal{A}
$$


where $\mathcal{A} \in R_{+}^{N} \times R^{N}$ is the set of all possible rate/bandwidth allocations to the network's users and the taxes (resp. subsidies) paid (resp. received) by the users. We call $\pi$ the solution of the centralized unicast service provisioning problem.

\section{B. The decentralized problem with strategic users}

We consider the network model of the previous section with the following assumptions on its information structure.

- (A1): Each user knows only his own utility; this utility is his own private information.

- (A2): Each user behaves strategically, that is, each user is not a price-taker. The users's objective is to maximize its own utility function.

- (A3): The network manager knows the topology and resources of the network. This knowledge is the manager's private information. The network manager is not a profit-maker (i.e. he does not have a utility function).

- (A4): The network manager receives requests for service from the network users. Based on these requests, he announces to each user $i, i \in \mathcal{N}$ :

1) The set of links that form user $i$ 's route, $\mathcal{R}_{i}$; that is, the network manager chooses the route for each user and this route remains fixed throughout the user's service.

2) The capacity of each link in $\mathcal{R}_{i}$.

- (A5) Based on the network manager's announcement, each strategic user competes for resources (bandwidth) at each link of his route with the other users in that link

From the above description it is clear that the information in the network is decentralized. Every user knows his own utility but does not know the other users' utilities or the network's topology and its resources. The network manager knows the network's topology and its resources, but does not know the users' utilities. It is also clear that the network manager (which is not profit maker) acts like an accountant who sets up the users' routes, specifies the users competing for resources/bandwidth at each link, collects the money from the users $i$ that pay tax (i.e. $t_{i}>0$ ) and distributes it to those users $j$ that receive money (i.e. $t_{i}<0$ ).

As a consequence of assumptions (A1)-(A5) we have at each link of the network a decentralized resource allocation problem which can be studied/analyzed within the context of

\footnotetext{
${ }^{2}$ During the play of the game at each link $l \in \mathbf{L}$, each user of link $l$ learns the set of the other users competing for bandwidth at $l$.
} 
implementation theory [33]. These decentralized resource allocation problems are not independent/decoupled, as the rate that each user receives at any link of his own route must be the same. This constraint is dictated by the nature of the unicast service provisioning problem and has a direct implication on the nature of the mechanism/game form we present in section III.

Under the above assumptions the objective is to determine a game form/mechanism which has the following properties,

- (P1). For each realization $\left(\mathbf{U}_{1}, \mathbf{U}_{2}, \cdots, \mathbf{U}_{N}, T\right) \in \mathcal{U}^{N} \times \mathbf{T}$, the Nash equilibria of the game induced by the mechanism result in allocations that are an optimal solution of the corresponding centralized problem Max; that is, the mechanism implements the map $\pi$ in Nash equilibria.

- (P2). The mechanism is individually rational, that is, for every realization

$$
\left(\mathbf{U}_{1}, \mathbf{U}_{2}, \cdots, \mathbf{U}_{N}, T\right) \in \mathcal{U}^{N} \times \mathbf{T}
$$

the network users voluntarily participate in the bandwidth allocation process.

- (P3). For every realization $\left(\mathbf{U}_{1}, \mathbf{U}_{2}, \cdots, \mathbf{U}_{N}, T\right) \in \mathcal{U}^{N} \times \mathbf{T}$ we have a balanced budget at every equilibrium point of the corresponding game form as well as off equilibrium.

Before proceeding with the specification of our mechanism, we comment on the appropriateness of Nash equilibrium as a solution concept for the decentralized problem under consideration. Nash equilibria describe strategic behavior in games of complete information. Since in our model, the network users do not know each others' utilities, for any realization $\left(\mathbf{U}_{1}, \mathbf{U}_{2}, \cdots, \mathbf{U}_{N}, T\right) \in$ $\mathcal{U}^{N} \times \mathbf{T}$ the resulting game is not a game of complete information. We can create a game of complete information by increasing the strategy space of the game following Maskin's approach [35]. Such an approach, however, would result in an infinite dimensional strategy space for the corresponding game. We do not follow Maskin's approach but we adopt the philosophy presented by Reichelstein and Reiter in [43] and Groves and Ledyard in [44]. Specifically:

”We interpret our analysis as applying to an unspecified (message exchange) process in which users grope their way to a stationary message and in which the Nash property is a necessary condition for stationarity”, Reichelstein and Reiter ( [43] pg. 664). 
" We do not suggest that each agent knows $\mathrm{e}^{3}$ when he computes $\boldsymbol{m}_{4}^{4}$.... We do suggest, however, that the 'complete information' Nash equilibrium game-theoretic equilibrium messages may be the possible equilibrium of the iterative process-that is, the stationary messages-just as the demand-equal-supply price is thought of the equilibrium of some unspecified market dynamic process.", Groves and Ledyard ( [44] pp. 69-70).

A philosophy similar to ours has also been adopted by Stoenescu and Ledyard in [30].

In the following two sections we present a mechanism/game form for the problem formulated in this section and prove that it possess properties P1 - P3 stated above.

\section{A Mechanism for Rate Allocation}

In section III-A we specify a mechanism/game form for the decentralized rate allocation problem formulated in section II] In section III-B, we discuss and interpret the components of the mechanism.

\section{A. Specification of the mechanism}

A game form/mechanism ( [33]) consists of two components $\mathcal{M}, f$. The component $\mathcal{M}$ denotes the users' message/strategy space. The component $f$ is the outcome function; $f$ defines for every message/strategy profile, the bandwidth/rate allocated to each user and the tax (subsidy) each user pays (receives).

For the decentralized resource allocation problem formulated in section II we propose a game form/mechanism the components of which we describe below.

Message space: The message/strategy space for user $i, i=1,2, \ldots, N$, is given by $\mathcal{M}_{i} \subset$ $R_{+}^{\left|\mathcal{R}_{i}\right|+1}$. Specifically, a message of user $i$ is of the form

$$
\mathbf{m}_{i}=\left(x_{i}, p_{i}^{l_{i_{1}}}, p_{i}^{l_{i_{2}}}, \cdots, p_{i}^{l_{i}\left|\mathcal{R}_{i}\right|}\right)
$$

where $0 \leq x_{i} \leq \min _{l \in \mathcal{R}_{i}} \mathbf{c}^{l}$ and $0 \leq p_{i}^{l_{i}} \leq M, k=1,2,, \cdots,\left|\mathcal{R}_{i}\right|, 0<M<\infty, M$ large, and $\left|\mathcal{R}_{i}\right|$ denotes the number of links along route $\mathcal{R}_{i}, i \in \mathcal{N}$. The component $x_{i}$ denotes the

\footnotetext{
${ }^{3}$ In our mechanism $\mathbf{e}=\left(\mathbf{U}_{1}, \mathbf{U}_{2}, \cdots, \mathbf{U}_{N}, T\right)$, that is, a realization of the users' utilities as well as of the topology and resources of the network.

${ }^{4}$ In our problem $\mathbf{m}_{i}$ is the strategy of user $i, i \in \mathcal{N}$.
} 
bandwidth/rate user $i$ requests at all the links of his route. The component $p_{i}^{l_{i}}, j=1,2, \cdots,\left|\mathcal{R}_{i}\right|$, denotes the price per unit of bandwidth user $i$ is willing to pay at link $l_{i_{j}}$ of his route.

As noted in section $\overline{I I-B}$, the nature of the unicast service provisioning problem dictates/requires that the bandwidth/rate allocated to any user $i, i \in \mathcal{N}$, must be the same at all links of his route. Thus, the nature of message $\mathbf{m}_{i}$ is a consequence of the above requirement.

Outcome Function: The outcome function $f$ is given by

$$
f: \mathcal{M}_{1} \times \mathcal{M}_{2} \times \cdots \times \mathcal{M}_{N} \rightarrow\left(\mathbb{R}_{+}^{N} \times \mathbb{R} \times \mathbb{R} \cdots \times \mathbb{R}\right)
$$

and is defined as follows. For any $\mathbf{m}:=\left(\mathbf{m}_{1}, \mathbf{m}_{2}, \cdots, \mathbf{m}_{N}\right) \in \mathcal{M}:=\mathcal{M}_{1} \times \mathcal{M}_{2} \times \cdots \times \mathcal{M}_{N}$,

$$
f(\mathbf{m})=f\left(\mathbf{m}_{1}, \mathbf{m}_{2}, \cdots, \mathbf{m}_{N}\right)=\left(x_{1}, x_{2}, \cdots, x_{N}, t_{1}, t_{2}, \cdots, t_{N}\right)
$$

where $x_{i}, i \in \mathcal{N}$, is the amount of bandwidth/rate allocated to user $i$ (this is equal to the amount of bandwidth user $i, i \in \mathcal{N}$, requests), and $t_{i}, i \in \mathcal{N}$, is determined by $t_{i}^{l}$, the tax (subsidy) user $i$ pays (receives) for link $l, l \in \mathcal{R}_{i}$, and by other additional subsidies $\mathcal{Q}^{i}$ that user $i$ may receive. We proceed now to specify $t_{i}^{l}, l \in \mathcal{R}_{i}$, and $\mathcal{Q}^{i}$ for every user $i \in \mathcal{N}$.

The tax $t_{i}^{l_{i_{j}}}, j=1,2, \cdots,\left|\mathcal{R}_{i}\right|, i \in \mathcal{N}$, is defined according to the number of users using link $l$. Let $\mathcal{G}^{l}$ denotes the set of users using link $l$ and let $\left|\mathcal{G}^{l}\right|$ denote the cardinality of $\mathcal{G}^{l}$. We consider three case 5

- CASE $1,\left|\mathcal{G}^{l}\right|=2$

Let $i, j \in \mathcal{G}^{l}$. Then,

$$
\begin{aligned}
& t_{i}^{l}=p_{j}^{l} x_{i}+\frac{\left(p_{i}^{l}-p_{j}^{l}\right)^{2}}{\alpha}-2 p_{j}^{l}\left(p_{i}^{l}-p_{j}^{l}\right)\left(\frac{x_{i}+x_{j}-\mathbf{c}^{l}}{\gamma}\right)+\frac{1\left\{x_{i}>0\right\} 1\left\{x_{i}+x_{j}-\mathbf{c}^{l}>0\right\}}{1-1\left\{x_{i}>0\right\} 1\left\{x_{i}+x_{j}-\mathbf{c}^{l}>0\right\}} \\
& t_{j}^{l}=p_{i}^{l} x_{j}^{l}+\frac{\left(p_{j}^{l}-p_{i}^{l}\right)^{2}}{\alpha}-2 p_{i}^{l}\left(p_{j}^{l}-p_{i}^{l}\right)\left(\frac{x_{i}+x_{j}-\mathbf{c}^{l}}{\gamma}\right)+\frac{1\left\{x_{j}>0\right\} 1\left\{x_{i}+x_{j}-\mathbf{c}^{l}>0\right\}}{1-1\left\{x_{j}>0\right\} 1\left\{x_{i}+x_{j}-\mathbf{c}^{l}>0\right\}}
\end{aligned}
$$

where $\alpha$ and $\gamma$ are positive constants that are sufficiently large and, the function $1\{A\}$, used throughout the paper, is defined as follows

$$
1\{A\}= \begin{cases}1-\epsilon & \text { if } A \text { holds } \\ 0 & \text { otherwise }\end{cases}
$$

${ }^{5}$ We consider only the cases where $\left|\mathcal{G}^{l}\right| \geq 2$. If $\left|\mathcal{G}^{l}\right|=1$ and $i \in \mathcal{G}^{l}$, then $t_{i}^{l}=0 \cdot 1\left\{x_{i} \leq \mathbf{c}^{l}\right\}+\frac{1\left\{x_{i}>\mathbf{c}^{l}\right\}}{1-1\left\{x_{i}>\mathbf{c}^{l}\right\}}$ 
where $\epsilon$ is bigger than zero and sufficiently small 6 ; $\epsilon$ is chosen by the mechanism designer.

- CASE 2, $\left|\mathcal{G}^{l}\right|=3$

Let $i, j$ and $k \in \mathcal{G}^{l}$. Then

$$
\begin{aligned}
t_{i}^{l}= & P_{-i}^{l} x_{i}+\left(p_{i}^{l}-P_{-i}^{l}\right)^{2}-2 P_{-i}^{l}\left(p_{i}^{l}-P_{-i}^{l}\right)\left(\frac{\mathcal{E}_{-i}^{l}+x_{i}}{\gamma}\right) \\
& +\frac{1\left\{x_{i}>0\right\} 1\left\{x_{i}+x_{j}+x_{k}-\mathbf{c}^{l}>0\right\}}{1-1\left\{x_{i}>0\right\} 1\left\{x_{i}+x_{j}+x_{k}-\mathbf{c}^{l}>0\right\}}+\Omega_{i}^{l} \\
t_{j}^{l}= & P_{-j}^{l} x_{j}+\left(p_{j}^{l}-P_{-j}^{l}\right)^{2}-2 P_{-j}^{l}\left(p_{j}^{l}-P_{-j}^{l}\right)\left(\frac{\mathcal{E}_{-j}^{l}+x_{j}}{\gamma}\right) \\
& +\frac{1\left\{x_{j}>0\right\} 1\left\{x_{i}+x_{j}+x_{k}-\mathbf{c}^{l}>0\right\}}{1-1\left\{x_{j}>0\right\} 1\left\{x_{i}+x_{j}+x_{k}-\mathbf{c}^{l}>0\right\}}+\Omega_{j}^{l} \\
t_{k}^{l}= & P_{-k}^{l} x_{k}+\left(p_{k}^{l}-P_{-k}^{l}\right)^{2}-2 P_{-k}^{l}\left(p_{k}^{l}-P_{-k}^{l}\right)\left(\frac{\mathcal{E}_{-k}^{l}+x_{k}}{\gamma}\right) \\
& +\frac{1\left\{x_{k}>0\right\} 1\left\{x_{i}+x_{j}+x_{k}-\mathbf{c}^{l}>0\right\}}{1-1\left\{x_{k}>0\right\} 1\left\{x_{i}+x_{j}+x_{k}-\mathbf{c}^{l}>0\right\}}+\Omega_{k}^{l}
\end{aligned}
$$

where,

$$
\begin{aligned}
& P_{-i}^{l}=\frac{p_{j}^{l}+p_{k}^{l}}{2}, P_{-j}^{l}=\frac{p_{k}^{l}+p_{i}^{l}}{2}, P_{-k}^{l}=\frac{p_{j}^{l}+p_{i}^{l}}{2} \\
& \mathcal{E}_{-i}^{l}=x_{j}+x_{k}-\mathbf{c}^{l}, \mathcal{E}_{-j}^{l}=x_{i}+x_{k}-\mathbf{c}^{l}, \mathcal{E}_{-k}^{l}=x_{i}+x_{j}-\mathbf{c}^{l} \\
& \mathcal{E}_{i}^{l}=2 x_{i}-\mathbf{c}^{l}, \mathcal{E}_{j}^{l}=2 x_{j}-\mathbf{c}^{l}, \mathcal{E}_{k}^{l}=2 x_{k}-\mathbf{c}^{l}
\end{aligned}
$$

and $\Omega_{i}^{l}$ is defined as

$$
\Omega_{i}^{l}=\frac{\sum_{\substack{r \in \mathcal{G}^{l} \\ r \neq i}} \sum_{\substack{s \in \mathcal{G}^{l} \\ s \neq i, r}}\left(2 p_{r}^{l} p_{s}^{l}\left(1+\frac{x_{r}}{\gamma}\right)-x_{r} p_{s}^{l}\right)}{\left(\left|\mathcal{G}^{l}\right|-1\right)\left(\left|\mathcal{G}^{l}\right|-2\right)}-\frac{\sum_{\substack{j \in \mathcal{G}^{l} \\ r \neq i}} p_{r}^{l 2}}{\left|\mathcal{G}^{l}\right|-1}-P_{-i}^{l 2}-2 \frac{\mathcal{E}_{-i}^{l} P_{-i}^{l}{ }^{2}}{\gamma} .
$$

The terms $\Omega_{j}^{l}$ and $\Omega_{k}^{l}$ are defined in a way similar to $\Omega_{i}^{l}$.

- CASE 3, $\left|\mathcal{G}^{l}\right|>3$

Let $i \in \mathcal{G}^{l} \subseteq \mathcal{N}$. Then,

${ }^{6}$ Therefore, when $\mathrm{A}$ and $\mathrm{B}$ (both) hold, then $\frac{1\{A\} 1\{B\}}{1-1\{A\} 1\{B\}} \approx \frac{1}{0^{+}}$is well defined and it becomes a large number. 


$$
\begin{aligned}
t_{i}^{l}= & P_{-i}^{l} x_{i}+\left(p_{i}^{l}-P_{-i}^{l}\right)^{2}-2 P_{-i}^{l}\left(p_{i}^{l}-P_{-i}^{l}\right)\left(\frac{\mathcal{E}_{-i}^{l}+x_{i}}{\gamma}\right)+\frac{1\left\{x_{i}>0\right\} 1\left\{\mathcal{E}_{-i}^{l}+x_{i}>0\right\}}{1-1\left\{x_{i}>0\right\} 1\left\{\mathcal{E}_{-i}^{l}+x_{i}>0\right\}} \\
& +\Phi_{i}^{l}
\end{aligned}
$$

where,

$$
P_{-i}^{l}=\frac{\sum_{\substack{j \in \mathcal{G}^{l} \\ j \neq i}} p_{j}^{l}}{\left|\mathcal{G}^{l}\right|-1}, \quad \mathcal{E}_{-i}^{l}=\sum_{\substack{j \in \mathcal{G}^{l} \\ j \neq i}} x_{j}-\mathbf{c}^{l}, \quad \mathcal{E}_{i}^{l}=\left(\left|\mathcal{G}^{l}\right|-1\right) x_{i}-\mathbf{c}^{l}
$$

and

$$
\begin{aligned}
\Phi_{i}^{l}= & \frac{\sum_{\substack{j \in \mathcal{G}^{l} \\
j \neq i}} \sum_{\substack{k \in \mathcal{G}^{l} \\
k \neq i, j}}\left(2 p_{j}^{l} p_{k}^{l}\left(1+\frac{x_{j}}{\gamma}\right)-x_{j} p_{k}^{l}\right)}{\left(\left|\mathcal{G}^{l}\right|-1\right)\left(\left|\mathcal{G}^{l}\right|-2\right)}+\frac{\sum_{\substack{j \in \mathcal{G}^{l} \\
j \neq i}} \sum_{\substack{k \in \mathcal{G}^{l} \\
k \neq i, j}} \sum_{\substack{r \in \mathcal{G}^{l} \\
r \neq i, j, k}} 2 p_{k}^{l}\left(p_{j}^{l} \mathcal{E}_{r}^{l}-x_{j} p_{r}^{l}\right)}{\gamma\left(\left|\mathcal{G}^{l}\right|-1\right)^{2}\left(\left|\mathcal{G}^{l}\right|-3\right)} \\
& +\frac{\sum_{\substack{j \in \mathcal{G}^{l} \\
j \neq i}} \sum_{\substack{k \in \mathcal{G}^{l} \\
k \neq i, j}} 2 p_{k}^{l}\left(p_{j}^{l} \mathcal{E}_{k}^{l}-x_{j} p_{k}^{l}\right)}{\gamma\left(\left|\mathcal{G}^{l}\right|-1\right)^{2}\left(\left|\mathcal{G}^{l}\right|-2\right)}-\frac{\sum_{\substack{j \in \mathcal{G}^{l} \\
j \neq i}} p_{j}^{l}{ }^{2}}{\left|\mathcal{G}^{l}\right|-1}-P_{-i}^{l 2}-2 \frac{\mathcal{E}_{-i}^{l} P_{-i}^{l 2}}{\gamma} .
\end{aligned}
$$

Next we specify additional subsidies $\mathcal{Q}^{i}$ that user $i, i \in \mathcal{N}$, may receive. For that matter we consider all links $l \in \mathbf{L}$ such that $\left|\mathcal{G}^{l}\right|=2$ or $\left|\mathcal{G}^{l}\right|=3$. For each link $l$, with $\left|\mathcal{G}^{l}\right|=2$ we define the quantity

$$
\begin{aligned}
\mathcal{Q}^{\left\{l:\left|\mathcal{G}^{l}\right|=2\right\}} & :=-2 \frac{\left(p_{i}^{l}-p_{j}^{l}\right)^{2}}{\alpha}-p_{j}^{l} x_{i}-p_{i}^{l}+x_{j}\left[2 p_{j}^{l}\left(p_{i}^{l}-p_{j}^{l}\right)+2 p_{i}^{l}\left(p_{j}^{l}-p_{i}^{l}\right)\right]\left(\frac{x_{i}+x_{j}-\mathbf{c}^{l}}{\gamma}\right) \\
& =o(1)-p_{j}^{l} x_{i}-p_{i}^{l} x_{j}
\end{aligned}
$$

for each link with $\left|\mathcal{G}^{l}\right|=3$ we define

$$
\mathcal{Q}^{\left\{l:\left|\mathcal{G}^{l}\right|=3\right\}}:=\frac{-2 P_{-i}^{l}{ }^{2} x_{-i}+2 P_{-i}^{l} p_{i}^{l} \mathcal{E}_{-i}^{l}-2 P_{-j}^{l}{ }^{2} x_{-j}+2 P_{-j}^{l} p_{j}^{l} \mathcal{E}_{-j}^{l}-2 P_{-k}^{l}{ }^{2} x_{-k}+2 P_{-k}^{l} p_{k}^{l} \mathcal{E}_{-k}^{l}}{\gamma} .
$$

Furthermore for each link $l \in \mathbf{L}$ where $\left|\mathcal{G}^{l}\right|=2$ or $\left|\mathcal{G}^{l}\right|=3$ the network manager chooses at random a user $k_{l} \notin \mathcal{G}^{l}$ and assigns the subsidy $\mathcal{Q}^{l}$ to user $k_{l}$. Let $l_{1}, l_{2}, \cdots, l_{r}$ be the set of links such that $\left|\mathcal{G}^{l_{i}}\right|=2$ or $3, i=1,2, \cdots, r$, and let $k_{l_{i}}, i=1,2, \cdots, r$, be the corresponding users that receive $\mathcal{Q}^{l_{i}}$. 
Based on the above, the tax (subsidy) paid (received) by user $j, j \in \mathcal{N}$, is the following. If $j \neq k_{l_{1}}, k_{l_{2}}, \cdots k_{l_{r}}$ then

$$
t_{j}=\sum_{l \in \mathcal{R}_{j}} t_{j}^{l}
$$

where for each $l \in \mathcal{R}_{j}, t_{j}^{l}$ is determined according to the cardinality of $\mathcal{G}^{l}$. If $j=k_{l_{i}}, i=$ $1,2, \cdots, r$, then

$$
t_{k_{l_{i}}}=\sum_{l \in \mathcal{R}_{k_{l_{i}}}} t_{k_{l_{i}}}^{l}+\mathcal{Q}^{l_{i}} .
$$

where $\mathcal{Q}^{l_{i}}$ is defined by (15) and (16).

Note that $\mathcal{Q}^{l_{i}}$ is not controlled by user $k_{l_{i}}$, that is, $\mathcal{Q}^{l_{i}}$ does not depend on user $k_{l_{i}}$ 's message/strategy. Thus, the presence (or absence) of $\mathcal{Q}^{l_{i}}$ does not influence the strategic behavior of user $k_{l_{i}}$. We have assumed here that the users $k_{l_{1}}, k_{l_{2}}, \cdots, k_{l_{r}}$, are distinct. Expressions similar to the above hold when the users $k_{l_{1}}, k_{l_{2}}, \cdots, k_{l_{r}}$ are not distinct.

Remark: For each link $l \in \mathcal{L}$ with $\left|\mathcal{G}^{l}\right|=2$ or 3 the network manager could equally divide the subsidy $\mathcal{Q}^{l}$ among all users not in $\mathcal{G}^{l}$ instead of randomly choosing one user $k \notin \mathcal{G}^{l}$. Any other division of the subsidy $\mathcal{Q}^{l}$ among users not in $\mathcal{G}^{l}$ would also work.

\section{B. Discussion/Interpretation of the Mechanism}

As pointed out in section $\Pi \mathrm{II}$, the design of a decentralized resource allocation mechanism has to achieve the following goals. (1) It must induce strategic users to voluntarily participate in the allocation process. (2) It must induce strategic users to follow its operational rules. (3) It must result in optimal allocations at all equilibria of the induced game. (4) It must result in a balanced budget at all equilibria and off equilibrium.

Since the designer of the mechanism can not alter the users' utility functions, $\mathbf{U}_{i}, i \in \mathcal{N}$, the only way it can achieve the aforementioned objectives is through the use of appropriate $\operatorname{tax}$ incentives/tax functions. At each link $l$, the tax incentive of our mechanism for user $i$ consists of three components $\Delta_{1}^{l}(i), \Delta_{2}^{l}(i)$ and $\Delta_{3}^{l}(i)$. We specify and interpret these components for Case 3 (Eq. (13)). Similar interpretations hold for Case 1 and Case 2. 
For Case 3 we have,

$$
t_{i}^{l}:=\Delta_{1}^{l}(i)+\Delta_{2}^{l}(i)+\Delta_{3}^{l}(i)
$$

where

$$
\begin{aligned}
& \Delta_{1}^{l}(i):=P_{-i}^{l} x_{i} \\
& \Delta_{2}^{l}(i):=\left(p_{i}^{l}-P_{-i}^{l}\right)^{2}-2 P_{-i}^{l}\left(p_{i}^{l}-P_{-i}^{l}\right)\left(\frac{\mathcal{E}_{-i}^{l}+x_{i}}{\gamma}\right)+\frac{1\left\{x_{i}>0\right\} 1\left\{\mathcal{E}_{-i}^{l}+x_{i}>0\right\}}{1-1\left\{x_{i}>0\right\} 1\left\{\mathcal{E}_{-i}^{l}+x_{i}>0\right\}} \\
& \Delta_{3}^{l}(i):=\Phi_{i}^{l}
\end{aligned}
$$

- $\Delta_{1}^{l}(i)$ specifies the amount user $i$ has to pay for the bandwidth it gets at link $l$. It is important to note that the price per unit of bandwidth that a user pays is determined by the message/proposal of the other users using the same link. Thus, a user does not control the price it pays per unit of the service it receives.

- $\Delta_{2}^{l}(i)$ provides the following incentives to the users of a link: (1) To bid/propose the same price per unit of bandwidth at that link (2) To collectively request a total bandwidth that does not exceed the capacity of the link. The incentive provided to all users to bid the same price per unit of bandwidth is described by the term $\left(p_{i}^{l}-P_{-i}^{l}\right)^{2}$. The incentive provided to all users to collectively request a total bandwidth that does not exceed the link's capacity is captured by the term

$$
\frac{1\left\{x_{i}>0\right\} 1\left\{\mathcal{E}_{-i}^{l}+x_{i}>0\right\}}{1-1\left\{x_{i}>0\right\} 1\left\{\mathcal{E}_{-i}^{l}+x_{i}>0\right\}} .
$$

Note that a user is very heavily penalized if it requests a nonzero bandwidth, and, collectively, all the users of the link request a total bandwidth that exceeds the link's capacity. A joint incentive provided to all users to bid the same price per unit of bandwidth and to utilize the total capacity of the link is captured by the term

$$
2 P_{-i}\left(p_{i}^{l}-P_{-i}^{l}\right)\left(\frac{\mathcal{E}_{-i}^{l}+x_{i}}{\gamma}\right)
$$

- $\Delta_{3}^{l}(i)$, The goal of this component is to lead to a balanced budget. That is,

$$
\sum_{i \in \mathcal{G}^{l}}\left[\Delta_{1}^{l}(i)+\Delta_{2}^{l}(i)\right] \neq 0
$$


but,

$$
\sum_{i \in \mathcal{G}^{l}}\left[\Delta_{1}^{l}(i)+\Delta_{2}^{l}(i)+\Delta_{3}^{l}(i)\right]=0
$$

Note that, $\Delta_{3}^{l}(i)$ is not controlled by user $i$ 's messages (simply because there is no term in $\Delta_{3}^{l}(i)$ under the control of user $i$ ), so $\Delta_{3}^{l}(i)$ does not have any influence on the strategic behavior of the user.

As indicated in (26), when the number of users at link $l \in \mathbf{L}$ is larger than three, i.e. $\left|\mathcal{G}^{l}\right|>3$, the mechanism is budget-balanced at that link, that is $\sum_{i \in \mathcal{G}^{l}} t_{i}^{l}=0$. When $\left|\mathcal{G}^{l}\right|=2,3$ the mechanism is not budget balanced at link $l$. The amount $\mathcal{Q}^{l}=-\sum_{\substack{i \in \mathcal{G}^{l} \\\left|\mathcal{G}^{l}\right|=2,3}} t_{i}^{l}$, is given as subsidy to a randomly chosen user, say $j$, that does not compete for resources at link $l$. Such money transfers results in an overall balanced budget, and are always possible whenever $N>3$. Furthermore, the money transfered to user $j$ does not alter $j^{\prime}$ s strategic behavior since $\mathcal{Q}^{l}$ does not depend on user $j^{\prime}$ s strategy. The existence of the term $\mathcal{Q}_{l_{j}}$ in the tax function couples the games that are taking place at various links of the network. The presence of $\mathcal{Q}^{l_{j}}$ implies that the designer of the mechanism must not consider links individually; for the allocation of resources at certain links (specially those links $l$ with $\left|\mathcal{G}^{l}\right|=2,3$ ) the design must consider network users that do not compete for resources in those links.

\section{Properties of the Mechanism}

We prove that the mechanism proposed in section III has the following properties: (P1) It implements the solution of Problem Max in Nash equilibria. (P2) It is individually rational. (P3) It is budget-balanced at every feasible allocation, that is the mechanism is budget-balanced at allocations corresponding to all $\mathrm{NE}$ messages as well as those corresponding to off-equilibrium messages. We also prove the existence of $\mathrm{NE}$ of the game induced by the mechanism and characterized all of them.

We establish the above properties by proceeding as follows. First we prove that all Nash equilibria of the game induced by the game form/mechanism of section [III result in feasible solutions of the centralized problem Max, (Theorem 11). Then, we show that network users voluntarily participate in the allocation process. We do this by showing that the allocations they receive at all Nash equilibria of the game induced by the game form of section [II are weakly 
preferred to the $(0,0)$ allocation they receive when they do not participate in the allocation process (Theorem 4). Afterwards, we establish that the mechanism is budget-balanced at all Nash equilibria; we also prove that the mechanism is budget-balanced off equilibrium (Lemma 22. Finally, we show that the mechanism implements in Nash equilibria the solution of the centralized allocation problem Max (Theorem 5).

We present the proofs of the following theorems and lemmas in the Appendix.

Theorem 1. (FeAsibiLity): If $\boldsymbol{m}^{*}=\left(\boldsymbol{x}^{*}, \boldsymbol{p}^{*}\right)$ is a NE point of the game induced by the game form and the users' utility(outcome) functions presented in section [II] then the allocation $\mathbf{x}^{*}$ is a feasible solution of Problem Max.

The following lemma presents some key properties of NE prices and rates.

Lemma 2. Let $\boldsymbol{m}^{*}=\left(\boldsymbol{x}^{*}, \boldsymbol{p}^{*}\right)$ be a $N E$. Then for every $l \in \boldsymbol{L}$ and $i \in \mathcal{G}^{l}$, we have,

$$
\begin{aligned}
p_{i}^{* l}=p_{j}^{* l}=P_{-i}^{* l}: & =p^{* l}, \\
p^{* l}\left(\frac{\mathcal{E}^{* l}}{\gamma}\right) & =0, \\
\left.\frac{\partial t_{i}^{l}}{\partial x_{i}}\right|_{m=m^{*}} & =p^{* l},
\end{aligned}
$$

where $\mathcal{E}^{* l}=\sum_{i \in \mathcal{G}^{l}} x_{i}^{*}-\boldsymbol{c}^{l}$.

An immediate consequence of Lemma 2 is the following. At every NE point $\mathbf{m}^{*}$ of the game induced by the mechanism the tax function has the following form,

$$
t_{i}^{l}\left(\mathbf{m}^{*}\right)= \begin{cases}p^{* l} x_{i}^{*} & \text { if }\left|\mathcal{G}^{l}\right|=2 ; \\ p^{* l}\left(x_{i}^{*}-x_{-i}^{*}\right)+\frac{\left(p^{* l}\right)^{2}\left(\mathbf{c}^{l}-\mathcal{E}_{-i}^{* l}\right)}{\gamma} & \text { if }\left|\mathcal{G}^{l}\right|=3 ; \\ p^{* l}\left(x_{i}^{*}-x_{-i}^{*}\right) & \text { if }\left|\mathcal{G}^{l}\right|>3 .\end{cases}
$$

Thus, by (15), (16), (17), (18) and Lemma 2 we have,

$$
t_{i}\left(\mathbf{m}^{*}\right)=\sum_{l \in \mathcal{R}_{i}} t_{i}^{l}\left(\mathbf{m}^{*}\right),
$$

for $i \neq k_{l_{1}}, k_{l_{2}}, \cdots, k_{l_{r}}$, (cf section IIII), and for $i=k_{l_{j}}, j=1,2, \cdots, r$, 


$$
t_{k_{l_{j}}}\left(\mathbf{m}^{*}\right)=\mathcal{Q}^{* l_{j}}+\sum_{l \in \mathcal{R}_{k_{l_{j}}}} t_{k_{l_{j}}}^{l}\left(\mathbf{m}^{*}\right) .
$$

In the following lemma, we prove that the proposed mechanism is always budget balanced.

Lemma 3. The proposed mechanism/game form is always budget balanced at every feasible allocation. That is, the mechanism is budget-balanced at all allocations corresponding to NE messages as well as at messages that are off equilibrium.

The next result asserts that the mechanism/game form proposed in section III is individually rational.

Theorem 4. (INDIVIDUAL RATIONALITY): The game form specified in section III is individually rational, that is at every NE of the game induced by the mechanism the corresponding allocation $\left(\mathbf{x}^{*}, \boldsymbol{t}^{*}\right)$ is weakly preferred by all users to the initial allocation $(0,0)$.

Finally, we prove that the mechanism of section III implements in NE the correspondence $\pi$ defined by the solution of Problem Max.

Theorem 5. (NASh Implementation): Consider any $N E \boldsymbol{m}^{*}$ of the game induced by the mechanism of section III Then, the allocation $\left(\mathbf{x}^{*}, \boldsymbol{t}^{*}\right)$ corresponding to $\boldsymbol{m}^{*}$ is an optimal solution of the centralized problem Max.

\section{EXISTENCE AND CHARACTERIZATION OF THE NASH EQUILIBRIA:}

So far, we have assumed the existence of NE of the game induced by the proposed game form/mechanism. In the following theorem, we prove that NE exist (recall the interpretation of NE we have given at the end of section III and characterize all of them.

Theorem 6. Let $\left(x_{1}^{*}, x_{2}^{*}, \cdots, x_{N}^{*}\right)$ be an optimal solution of Problem Max and $\lambda^{* l}, l \in \boldsymbol{L}$, be the corresponding Lagrange multipliers of the Karush-Kuhn-Tucker (KKT) conditions. Then

$$
\boldsymbol{m}^{*}:=\left(x_{1}^{*}, x_{2}^{*}, \cdots, x_{N}^{*}, p^{* l_{1}}, p^{* l_{2}}, \cdots, p^{* l_{L}}\right)
$$

with $p^{* l}=\lambda^{* l}, l \in \boldsymbol{L}$ is a NE of the game induced by the proposed game form. 


\section{IMPLEMENTATION OF THE DECENRALIZED MECHANISM}

First, we discuss how the mechanism specified by the game form of section III can be implemented at equilibrium. Then, we address the computation of the NE of the game induced by the game form of this paper.

We present one way of implementing the proposed mechanism at equilibrium. Consider an arbitrary link $l$ of the network. The users of that link communicate their equilibrium messages to one another and to the network manager. The network manager determines the rate and tax (or subsidy) of each user and announces this information to the user. The users $i, i \in \mathcal{N}$ with $\operatorname{tax} t_{i}^{l}>0$ pay the amount $t_{i}^{l}$ to the network manager; the network manager redistributes the amount of money it receives to the users $j \in \mathcal{N}$ with $t_{j}^{l}<0$. In the situation where the number of users in the link is equal to two (resp. three) the network manager chooses randomly a user not using that link to whom it gives the subsidy $\mathcal{Q}^{*\left\{l:\left|\mathcal{G}^{l}\right|=2\right\}}$ (resp. $\mathcal{Q}^{*\left\{l:\left|\mathcal{G}^{l}\right|=3\right\}}$ ) defined by (15) (resp. (16)). The above described process is repeated/takes place at every network link. This process implements the mechanism described in the paper at equilibrium.

Even though for the specific form of the tax we have provided a complete characterization of the NE of the game induced by the game form proposed in the paper, currently we do not have an algorithm for the computation of these equilibria. Based on preliminary investigation, we believe that best response algorithms do not, in general, guarantee convergence to NE equilibria, because the game induced by the game form proposed in this paper is not supermodular (Ch. 12, [22]) (due to the capacity constraint present at each link). Thus, the algorithmic computation of the NE of the game induced by the game form proposed in the paper remains as an open problem.

\section{AN EXTENSION}

So far we required that the users' utility functions be concave. We now weaken this requirement; we assume that the users' utilities are quasi-concave. We consider the game form proposed in section III. By repeating the arguments of Theorem 1, Lemma 2, Lemma 3 and Theorem 4 we can show that: every NE of the game induced by the game form is feasible; the game form/mechanism is individually rational and budget-balanced at all feasible allocations, i.e. at every NE and off equilibrium. In the following theorem we prove that every NE of the game induced by the proposed game form results in a Walrasian Equilibrium (WE) [12]. 
Theorem 7. Consider the game $\left(\mathcal{M}, f, \mathcal{V}_{i}, i=1,2, \cdots, N\right)$, induced by the game form of section [II] with continuous and quasi-concave utilities $\boldsymbol{U}_{i}, i \in \mathcal{N}$. Then, every $N E \boldsymbol{m}^{*}$ of this game results in a Walrasian equilibrium, hence a Pareto optimal allocation $\left(\boldsymbol{x}^{*}, \boldsymbol{t}^{*}\right)$.

\section{CONCLUSION}

We have proposed a mechanism for rate/bandwidth allocation in unicast service provisioning and performed an equilibrium analysis of the mechanism. We discovered that when the users' utilities are concave, the mechanism possesses the following properties: (i) It implements in Nash equilibria the solution of the corresponding centralized rate allocation problem. (ii) It is individually rational. (iii) It is budget-balanced at all feasible allocations, i.e. at all Nash equilibria of the game induced by the mechanism/game form as well as off equilibrium. When the users' utilities are quasi-concave the proposed mechanism possesses properties (ii) and (iii) stated above. Moreover, every Nash equilibrium of the game induced by the proposed mechanism results in a Walrasian equilibrium, hence a Pareto optimal allocation.

The development of algorithms that guarantee convergence to Nash equilibria of the game corresponding to the mechanism of this paper is an important problem. We have not studied this problem in detail. Preliminary investigation indicates that best response algorithms do not guarantee convergence to Nash equilibria, since the game induced by the game form developed in this paper is not supermodular (due to the capacity constraint present in the game played at each link).

\section{Acknowledgments}

This research was supported in part by NSF Grant CCR-0325571. The authors are grateful to $\mathrm{M}$. Liu for useful discussions.

\section{REFERENCES}

[1] R. Cocchi, D. Estrin, S. Shenker, and L. Zhang, "Pricing in computer networks: motivation, formulation and example," IEEE/ACM Transaction on Networking, vol. 1, no. 6, pp. 614-627, 1993.

[2] G. de Veciana and R. Baldick, "Resource allocation in multiservice networks via pricing," Computer Networks and ISDN Systems, no. 30, pp. 951-962, 1998.

[3] S. Deb and R. Srikant, "Congestion control for fair resource allocation in networks with multicast flows," IEEE/ACM Transactions on Networking, vol. 12, no. 2, pp. 261-273, 2004. 
[4] H. Jiang and S. Jordan, "The role of price in the connection establishment process," European Transactions on Telecommunications, vol. 6, no. 4, pp. 421-429, 1995.

[5] S. Jordan and H. Jiang, "Connection establishment in high speed networks," IEEE Journal of Selected Areas in Communications, vol. 13, no. 7, pp. 1150-1161, 1995.

[6] F. Kelly, "On tariffs, policing and admission control for multiservice networks," Operations Research Letters, vol. 15, pp. $1-9,1994$.

[7] — - "Charging and rate control for elastic traffic," Euro. Trans. on Telecommunication, pp. 33-37, 1997.

[8] F. Kelly, A. Maulloo, and D. Tan, "Rate control for communication networks: shadow prices, proportional fairness and stability," Operational Research Society, pp. 237-252, 1998.

[9] S. Low and P. Varaiya, "A new approach to service provisioning in atm networks," IEEE/ACM Transactions on Networking, pp. 547-553, 1993.

[10] J. MacKie-Mason and H. Varian, "Pricing congestible network resources," Journal of Selected Areas in Communications, pp. 1141-1149, 1995.

[11] _- "Some faqs about usage-based pricing," Computer Networks and ISDN Systems, vol. 13, no. 7, pp. 257-265, 1995.

[12] A. Mas-colell, M. Whinston, and J. Green, Microeconomics Theory. Oxford University Press, 1995.

[13] L. Murphy and J. Murphy, "Bandwidth allocation by pricing in atm networks," In Proceedings of the International Teletraffic Congress, 1994.

[14] L. Murphy, J. Murphy, and E. Posner, "Distributed pricing for embedded atm networks," In Proceedings of the International Teletraffic Congress, 1994.

[15] C. Parris and D. Ferrari, "resource based pricing policy for real-time channels in a packet-switching network," TR-92-018, International Computer Science Institute, Berkeley CA, 1992.

[16] C. Parris, S. Keshav, and D. Ferrari, "A framework for the study of pricing in integrated networks," TR-92-016, International Computer Science Institute, Berkeley CA, 1992.

[17] S. Reichelstein, Information and incentives in economic organizations. Ph.D. dissertation, Northwestern University, Evanston, IL, 1984.

[18] S. Sharma and D. Teneketzis, "Game forms with minimal strategy spaces," Telecommunication Systems Journal, to appear.

[19] P. Thomas and D. Teneketzis, "An approach to service provisioning with quality of service requirements in atm networks," Journal of High Speed Networks, vol. 6, no. 4, pp. 263-291, 1997.

[20] P. Thomas, D. Teneketzis, and J. MacKie-Mason, "A market-based approach to optimal resource allocation in integratedservices connection-oriented networks," Operations Research, vol. 50, no. 5, pp. 603-616, 2002.

[21] Q. Wang, J. Peha, and M. Sirbu, "Optimal pricing for integrated-services networks," Internet Economics, pp. 353-376, 1997.

[22] D. Fudenberg and J. Tirole, Game Theory. MIT Press, 1991.

[23] B. Hajek and S. Yang, "Strategic buyers in a sum-bid game for flat networks," manuscript, 2004.

[24] R. Johari and J. Tsitsiklis, "Efficiency loss in a network resource allocation game," Mathematics of operation research, vol. 29, no. 3, pp. 407-435, 2004.

[25] — - "Communication requirement of vcg-like mechanisms in convex environments," Proc. of the 43rd Annual Allerton Conference on Communication, Control and Computing, 2005.

[26] A. Lazar and N. Semret, "The progressive second price auction mechanism for network resource sharing," Proc. Int. Symp. on Dynamic Games and Applications, 1997. 
[27] _ _ "Design and analysis of the progressive second price auction for network bandwidth sharing," Telecommunication Systems - Special issue on Network Economics, 1999.

[28] R. Maheswaren and T. Basar, "Nash equilibrium and decenteralized negotiation in auctioning divisible resources," J. Group Decision and Negotiation, 2003.

[29] —- "Social welfare of selfish agents: Motivating efficiency for divisible resources," Proc. Control and Decision Conferences (CDC), pp. 361-395, 2004.

[30] T. Stoenescu and J. Ledyard, "Nash implementation for resource allocation network problems with production," manuscript, 2008.

[31] S. Yang and B. Hajek, "Vcg-kelly mechanisms for allocation of divisible goods: Adapting vcg mechanisms to onedimensional signals," IEEE. J. Selected Areas of Communications, pp. 1237-1243, 2007.

[32] S. Sharma, A Mechanism design approach to decentralized resource allocation in wireless and large-scale networks: Realization and Implementation. Ph.D. dissertation, University of Michigan, Ann Arbor, MI, 2009.

[33] L. Hurwicz and S. Reiter, Designing Economics Mechanisms. Cambridge University Press, 2006.

[34] S. R. Williams, Communication in Mechanism Design: A Differential Approach. Cambridge University Press, 2008.

[35] E. Maskin, "The theory of implementation in nash equilibrium: A survey," Social Goals and Social Organization: Ch. 6, pp. 173-204, 1985.

[36] T. Stoenescu and D. Teneketzis, "Decentralized resource allocation mechanisms in networks: Realization and implementation," Advances in Control, Communication Networks, and Transportation Systems, in Honor of Pravin Varaiya, pp. 225-266, 2005.

[37] A. Dimakis, R. Jain, and J. Walrand, "Mechanisms for efficient allocation in divisible capacity networks."

[38] R. Jain and J. Walrand, "An efficient nash-implementation mechanism for divisible resource allocation," Automatica, vol. 46, no. 8 , pp. $1276-1283$.

[39] W. Vickrey, "Counterspeculation, auctions, and sealed tenders," J. of Finance, pp. 8-37, 1961.

[40] E. Clarke, "Multipart pricing of public good," Public Choice, vol. 2, pp. 19-33, 1971.

[41] T. Groves, "Incentive in teams," vol. 41, pp. 617-631, 1973.

[42] S. Yang and B. Hajek, "Revenue and stability of a mechanism for efficient allocation of a divisible good," submitted to Journal of Franklin Institute, 2006.

[43] S. Reichelstein and S. Reiter, "Game forms with minimal strategy spaces," Econometrica, pp. 661-692, 1988.

[44] T. Groves and J. Ledyards, "Incentive compatibility since 1972," Information, Incentives, and Economics Mechanisms, Essays in Honor of Leonid Hurwics, pp. 48-111, 1987.

[45] A. Kakhbod and D. Teneketzis, "An efficient game form for unicast service provisioning," arXiv:0910.5502, 2009.

[46] R. Bracwell, The Fourier Transform and Its Applications. New York: McGraw-Hill, 1999.

[47] S. Boyd and L. Vandenberghe, Convex Optimization. Cambridge University Press, 2004.

\section{APPENDIX}

Proof of Theorem 1]: By the construction of the mechanism $x_{i}^{*} \geq 0$ for all $i \in \mathcal{N}$. Suppose that $\mathbf{x}^{*}=\left(x_{1}^{*}, \cdots, x_{N}^{*}\right)$ is such that the capacity constraint is violated at some link $l$ and $x_{j}^{*}>0$ (i.e. user $j$ will be heavily charged because $\frac{1\left\{x_{j}^{*}>0\right\} 1\left\{\mathcal{E}_{-j}^{* l}+x_{j}^{*}>0\right\}}{1-1\left\{x_{j}^{*}>0\right\} 1\left\{\mathcal{E}_{-j}^{* l}+x_{j}^{*}>0\right\}} \approx \frac{1}{0^{+}}$which is a large number). 
Now, Consider $x_{j}$ such that: (i) either $x_{j}>0$ and $\sum_{\substack{k \in \mathcal{G}^{l} \\ k \neq j}} x_{k}^{*}+x_{j} \leq \mathbf{c}^{\mathbf{l}}$; or (ii) $x_{j}=0$. Then, $\frac{1\left\{x_{j}>0\right\} 1\left\{\mathcal{E}_{-j}^{* l}+x_{j}>0\right\}}{1-1\left\{x_{j}>0\right\} 1\left\{\mathcal{E}_{-j}^{* l}+x_{j}>0\right\}}=0$, therefore,

$$
\mathcal{V}_{j}\left(\mathbf{m}_{j}, \mathbf{m}_{-j}^{*}\right)>\mathcal{V}_{j}\left(\mathbf{m}_{j}^{*}, \mathbf{m}_{-j}^{*}\right)
$$

and (33) contradicts the fact that $\mathbf{m}^{*}=\left(\mathbf{m}_{j}^{*}, \mathbf{m}_{-j}^{*}\right)$ is a NE. Consequently, $\mathbf{x}^{*}$ is a feasible allocation of problem Max.

Proof of Lemma 2. We prove this lemma by considering the case $\left|\mathcal{G}^{l}\right|>3$. The cases $\left|\mathcal{G}^{l}\right|=2$ and $\left|\mathcal{G}^{l}\right|=3$ can be proved similarly. A detailed proof for $\left|\mathcal{G}^{l}\right|=2$ and $\left|\mathcal{G}^{l}\right|=3$ is available in [45].

Consider user $i \in \mathcal{G}^{l}\left(\left|\mathcal{G}^{l}\right|>3\right.$ ). Since user $i$ does not control $\Phi_{i}^{l}$ defined by (14]), (i.e. $\Phi_{i}^{l}$ does not depend on $x_{i}$ and $p_{i}^{l}$ ),

$$
\frac{\partial \Phi_{i}^{l}}{\partial x_{i}}=\frac{\partial \Phi_{i}^{l}}{\partial p_{i}^{l}}=0
$$

Eq. (13) along with (34) imply

$$
\left.\frac{\partial t_{i}^{l}}{\partial p_{i}^{l}}\right|_{\mathbf{m}=\mathbf{m}^{*}}=2\left[\left(p_{i}^{* l}-P_{-i}^{* l}\right)-P_{-i}^{* l}\left(\frac{\mathcal{E}_{-i}^{* l}+x_{i}^{*}}{\gamma}\right)\right]=0 .
$$

Summing Eq. (35) over all $i \in \mathcal{G}^{l}$, we get,

$$
\left.\sum_{i \in \mathcal{G}^{l}} \frac{\partial t_{i}^{l}}{\partial p_{i}^{l}}\right|_{\mathbf{m}=\mathbf{m}^{*}}=\sum_{i \in \mathcal{G}^{l}}\left[\left(p_{i}^{* l}-P_{-i}^{* l}\right)-P_{-i}^{* l}\left(\frac{\mathcal{E}_{-i}^{* l}+x_{i}^{*}}{\gamma}\right)\right]=\sum_{i \in \mathcal{G}^{l}}-P_{-i}^{* l}\left(\frac{\mathcal{E}_{-i}^{* l}+x_{i}^{*}}{\gamma}\right)=0,
$$

which, because of Theorem 1 and the positivity of prices, implies

$$
-P_{-i}^{* l}\left(\frac{\mathcal{E}_{-i}^{* l}+x_{i}^{*}}{\gamma}\right)=0 .
$$

for every $i \in \mathcal{G}^{l}$. Then Eq. (37) gives

$$
p_{i}^{* l}=P_{-i}^{* l} .
$$

for all $i \in \mathcal{G}^{l}$. From Eqs. (37) and (38) it follows that, 


$$
\begin{aligned}
& p^{* l}\left(\frac{\mathcal{E}^{* l}}{\gamma}\right)=0, \\
& p_{i}^{* l}=p_{j}^{* l}=P_{-i}^{* l}=p^{* l} .
\end{aligned}
$$

Eqs. (39) and (40) along with (13) give

$$
\left.\frac{\partial t_{i}^{l}}{\partial x_{i}}\right|_{\mathbf{m}=\mathbf{m}^{*}}=p^{* l}
$$

By (40), (39) and (41) $)^{7}$ the proof is complete.

Proof of Lemma 3: Equation (30) together with (31) and 32) imply that $\sum_{l \in \mathbf{L}} \sum_{i \in \mathcal{G}^{l}} t_{i}^{* l}=$ 0 . Now, we prove that the proposed mechanism is also budget balanced off equilibrium. First we show that, for every $l \in \mathbf{L}$ where $\left|\mathcal{G}^{l}\right|>3$

$$
\sum_{i \in \mathcal{G}^{l},\left|\mathcal{G}^{l}\right|>3} t_{i}^{l}=0 .
$$

By a little algebra we can show the following equalities,

$$
\begin{aligned}
& \sum_{i \in \mathcal{G}^{l}} p_{i}^{l^{2}}=\sum_{i \in \mathcal{G}^{l}}\left[\frac{\sum_{\substack{j \in \mathcal{G}^{l} \\
j \neq i}}^{p_{j}^{l}}{ }^{2}}{\left|\mathcal{G}^{l}\right|-1}\right]
\end{aligned}
$$

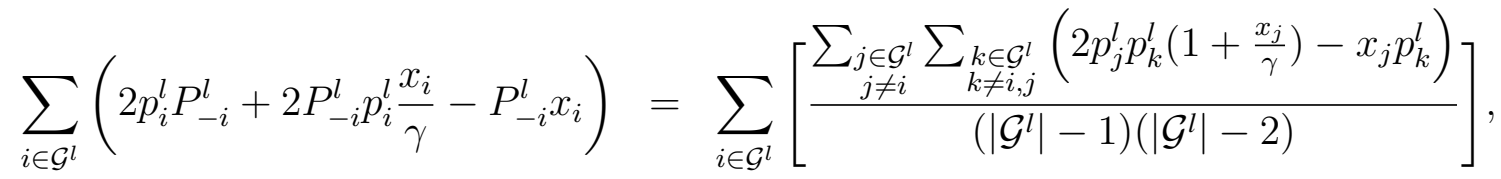

$$
\begin{aligned}
& \sum_{i \in \mathcal{G}^{l}} P_{-i}^{l} p_{i}^{l} \frac{\mathcal{E}_{-i}^{l}}{\gamma}=\sum_{i \in \mathcal{G}^{l}}\left[\frac{\sum_{\substack{j \in \mathcal{G}^{l} \\
j \neq i}} \sum_{\substack{k \in \mathcal{G}^{l} \\
k \neq i, j}} \sum_{\substack{r \in \mathcal{G}^{l} \\
r \neq i, j, k}} 2 p_{k}^{l} p_{j}^{l} \mathcal{E}_{r}^{l}}{\gamma\left(\left|\mathcal{G}^{l}\right|-1\right)^{2}\left(\left|\mathcal{G}^{l}\right|-3\right)}+\frac{\sum_{\substack{j \in \mathcal{G}^{l} \\
j \neq i}} \sum_{\substack{k \in \mathcal{G}^{l} \\
k \neq i, j}} 2 p_{k}^{l} p_{j}^{l} \mathcal{E}_{k}^{l}}{\gamma\left(\left|\mathcal{G}^{l}\right|-1\right)^{2}\left(\left|\mathcal{G}^{l}\right|-2\right)}\right], \\
& \sum_{i \in \mathcal{G}^{l}} P_{-i}^{l}{ }^{2} \frac{x_{i}}{\gamma}=\sum_{i \in \mathcal{G}^{l}}\left[\frac{\sum_{\substack{j \in \mathcal{G}^{l} \\
j \neq i}} \sum_{\substack{k \in \mathcal{G}^{l} \\
k \neq i, j}} \sum_{\substack{r \in \mathcal{G}^{l} \\
r \neq i, j, k}} x_{j} p_{r}^{l}}{\gamma\left(\left|\mathcal{G}^{l}\right|-1\right)^{2}\left(\left|\mathcal{G}^{l}\right|-3\right)}+\frac{\sum_{\substack{j \in \mathcal{G}^{l} \\
j \neq i}} \sum_{\substack{k \in \mathcal{G}^{l} \\
k \neq i, j}} x_{j} p_{k}^{l}}{\gamma\left(\left|\mathcal{G}^{l}\right|-1\right)^{2}\left(\left|\mathcal{G}^{l}\right|-2\right)}\right] .
\end{aligned}
$$

\footnotetext{
${ }^{7}$ Note that, since the derivative of an indicator function is a Dirac delta function ( [46], p. 94), to have a well defined derivative of $t_{i}$ with respect to $x_{i}$ at the boundary, i.e., when $\sum_{i \in \mathcal{G}^{l}} x_{i}=\mathbf{c}^{l}$, the differentiation is from the left. This observation holds throughout the proofs appearing in this Appendix.
} 
From (43)-(46) we conclude that

$$
\sum_{i \in \mathcal{G}^{l}}\left[P_{-i}^{l} x_{i}+\left(p_{i}^{l}-P_{-i}^{l}\right)^{2}-2 P_{-i}^{l}\left(p_{i}^{l}-P_{-i}^{l}\right)\left(\frac{\mathcal{E}_{-i}^{l}+x_{i}}{\gamma}\right)\right]=-\sum_{i \in \mathcal{G}^{l}} \Phi_{i}^{l}
$$

Eq. (47) along with Eq. (13) imply that $\sum_{i \in \mathcal{G}^{l},\left|\mathcal{G}^{l}\right|>3} t_{i}^{l}=0$.

Next consider all links $l \in \mathbf{L}$ where $\left|\mathcal{G}^{l}\right|=2,3$. In accordance with the notation in section III. let these links be $l_{1}, l_{2}, \cdots, l_{r}$. Then, by the specification of the tax function (cf. section III-A, Eqs. (6)-(10) ) we obtain,

$$
\sum_{j=1}^{r}\left\{\left[t_{i_{l_{j, 1}}}^{l_{j}}+t_{i_{l_{j, 2}}}^{l_{j}}\right] 1\left\{\left|\mathcal{G}^{l_{j}}\right|=2\right\}+\left[t_{i_{l_{j, 1}}}^{l_{j}}+t_{i_{l_{j, 2}}}^{l_{j}}+t_{i_{l_{j, 3}}}^{l_{j}}\right] 1\left\{\left|\mathcal{G}^{l_{j}}\right|=3\right\}\right\}+\sum_{j=1}^{r} \mathcal{Q}^{l_{j}}=0
$$

where if $\left|\mathcal{G}^{l_{j}}\right|=2$ then $\left\{i_{l_{j, 1}}, i_{l_{j, 2}}\right\}=\mathcal{G}^{l_{j}}$ and if $\left|\mathcal{G}^{l_{j}}\right|=3$ then $\left\{i_{l_{j, 1}}, i_{l_{j, 2}}, i_{l_{j, 3}}\right\}=\mathcal{G}^{l_{j}}, j=$ $1,2, \cdots, r$.

Finally note that,

$$
\sum_{i=1}^{N} t_{i}=\sum_{l \in \mathbf{L}:\left|\mathcal{G}^{l}\right|=2} \sum_{i \in \mathcal{G}^{l}} t_{i}^{l}+\sum_{l \in \mathbf{L}:\left|\mathcal{G}^{l}\right|=3} \sum_{i \in \mathcal{G}^{l}} t_{i}^{l}+\sum_{l \in \mathbf{L}:\left|\mathcal{G}^{l}\right|>3} \sum_{i \in \mathcal{G}^{l}} t_{i}^{l}+\sum_{j=1}^{r} \mathcal{Q}^{l_{j}}=0 .
$$

Proof of Theorem 4. We need to show that $\mathcal{V}_{i}\left(\mathbf{x}^{*}, t_{i}^{*}\right) \geq \mathcal{V}_{i}(0,0)=0$ for every $i \in \mathcal{N}$. By the property of NE it follows that

$$
\mathcal{V}_{i}\left(\mathbf{x}^{*}, t^{*}\right) \geq \mathcal{V}_{i}\left(\mathbf{x}_{-i}^{*}, x_{i}, t_{i}, t_{-i}^{*}\right) \quad \forall\left(x_{i}, \mathbf{t}_{i}\right)
$$

So, it is enough to find $\left(x_{i}, \mathbf{p}_{i}\right) \in \mathcal{M}_{i}$ such that

$$
\mathcal{V}_{i}\left(\mathbf{x}_{-i}^{*}, x_{i}, t_{i}, t_{-i}^{*}\right) \geq 0
$$

We set $x_{i}=0$ and examine the cases $\left|\mathcal{G}^{l}\right|=2,\left|\mathcal{G}^{l}\right|=3$ and $\left|\mathcal{G}^{l}\right|>3$, separately.

- CASE $1,\left|\mathcal{G}^{l}\right|=2$

With $x_{i}=0, p_{j}^{l}=p^{* l}$ and $x_{j}=x_{j}^{*}$, Eq. (6) defines the following function $\mathcal{F}_{2}\left(p_{i}^{l}\right)$ :

$$
\mathcal{F}_{2}\left(p_{i}^{l}\right):=\frac{\left(p_{i}^{l}-p^{* l}\right)^{2}}{\alpha}-2^{* l}\left(p_{i}^{l}-p^{* l}\right)\left(\frac{x_{j}^{*}-\mathbf{c}^{l}}{\gamma}\right)
$$


Clearly, at

$$
p_{i}^{l}=p^{* l}
$$

$\mathcal{F}_{2}\left(p^{* l}\right)=0$. Then, from Eq. (6) it follows that

$$
t_{i}^{l}\left(\mathbf{x}_{-i}^{*}, 0, \mathbf{p}_{-i}^{*}, p^{* l}\right)=0
$$

- CASE 2, $\left|\mathcal{G}^{l}\right|=3$

Denote by $i, j, k$ the users of link $l$. With $x_{i}=0, x_{j}=x_{j}^{*}, x_{k}=x_{k}^{*}$ and $p_{j}^{l}=p_{k}^{l}=p^{* l}$, Eq. (8) defines the following function $\mathcal{F}_{3}\left(p_{i}^{l}\right)$ :

$$
\begin{aligned}
\mathcal{F}_{3}\left(p_{i}^{l}\right) & :=\left(p_{i}^{l}-p^{* l}\right)^{2}-2 p^{* l}\left(p_{i}^{l}-p^{* l}\right)\left(\frac{x_{j}^{*}+x_{k}^{*}-\mathbf{c}^{l}}{\gamma}\right)+\Omega_{i}^{* l} \\
& =p_{i}^{l^{2}}-2 p_{i}^{l} p^{* l}\left(1+\frac{\mathcal{E}_{-i}^{* l}}{\gamma}\right)+p^{* l^{2}}\left(1+2 \frac{\mathcal{E}_{-i}^{* l}}{\gamma}\right)+\Omega_{i}^{* l}
\end{aligned}
$$

$\mathcal{F}_{3}\left(p_{i}^{l}\right)$ is a quadratic polynomial in $p_{i}^{l}$. Setting $\mathcal{F}_{3}\left(p_{i}^{l}\right)=0$ we obtain the root

$$
\wp_{i, 3}^{l}=p^{* l}\left(1+\frac{\mathcal{E}_{-i}^{* l}}{\gamma}\right)+\sqrt{\left(p^{* l} \frac{\mathcal{E}_{-i}^{* l}}{\gamma}\right)^{2}+x_{-i}^{*} p^{* l}+\frac{p^{* l^{2}}\left(\mathbf{c}^{l}-\mathcal{E}_{-i}^{* l}\right)}{\gamma}}
$$

Since by its definition $\gamma$ is sufficiently large, it follows from Eq. (55) that $\wp_{i, 3}^{l}>0$, i.e. $\wp_{i, 3}^{l}$ is a feasible price. Therefore, from Eq. (8) we obtain

$$
t_{i}^{l}\left(\mathbf{x}_{-i}^{*}, 0, \mathbf{p}_{-i}^{*}, \wp_{i, 3}^{l}\right)=0
$$

- CASE 3, $\left|\mathcal{G}^{l}\right|>3$

With $x_{i}=0, x_{j}=x_{j}^{*} \forall j \neq i, j \in \mathcal{G}^{l}, p_{j}^{l}=p^{* l}, j \in \mathcal{G}^{l}$, Eqs. (13) and (14) define (after a little algebra) the following function $\mathcal{F}_{>3}\left(p_{i}^{l}\right)$, 


$$
\begin{aligned}
\mathcal{F}_{>3}\left(p_{i}^{l}\right) & :=\left(p_{i}^{l}-p^{* l}\right)^{2}-2 p^{* l}\left(p_{i}^{l}-p^{* l}\right)\left(\frac{\mathcal{E}_{-i}^{* l}}{\gamma}\right)+\Phi_{i}^{* l} \\
& =p_{i}^{l^{2}}-2 p_{i}^{l} p^{* l}\left(1+\frac{\mathcal{E}_{-i}^{* l}}{\gamma}\right)+p^{* l^{2}}\left(1+2 \frac{\mathcal{E}_{-i}^{* l}}{\gamma}\right)-x_{-i}^{*} p^{* l}
\end{aligned}
$$

$\mathcal{F}_{>3}\left(p_{i}^{l}\right)$ is a quadratic polynomial in $p_{i}^{l}$. Setting $\mathcal{F}_{>3}\left(p_{i}^{l}\right)=0$ we obtain the root

$$
\wp_{i,>3}^{l}=p^{* l}\left(1+\frac{\mathcal{E}_{-i}^{* l}}{\gamma}\right)+\sqrt{\left(p^{* l} \frac{\mathcal{E}_{-i}^{* l}}{\gamma}\right)^{2}+x_{-i}^{*} p^{* l}}
$$

where

$$
x_{-i}^{*}:=\frac{\sum_{j \neq i} x_{j}^{*}}{\left|\mathcal{G}^{l}\right|-1} .
$$

Since by its definition $\gamma$ is sufficiently large, it follows from Eq. (58) that $\wp_{i,>3}^{l}>0$ (i.e. $\wp_{i,>3}^{l}$ is a feasible price). Therefore, from Eq. (13) we get

$$
t_{i}^{l}\left(\mathbf{x}_{-i}^{*}, 0, \mathbf{p}_{-i}^{*}, \wp_{i,>3}^{l}\right)=0 .
$$

Consequently, at $\mathbf{m}_{i}=\left(x_{i}, \mathbf{p}_{i}\right)=\left(0, p_{i}^{l_{i_{1}}}, p_{i}^{l_{i_{2}}}, \cdots, p_{i}^{l_{i}\left|\mathcal{R}_{i}\right|}\right)$, (where, $p_{i}^{l_{i_{k}}}, k=1,2, \cdots,\left|\mathcal{R}_{i}\right|$, are defined either by (52) or (55) or (58), depending on the cardinality $\left.\mathcal{G}^{l_{i}}, k=1,2, \cdots,\left|\mathcal{R}_{i}\right|\right)$, we obtain

$$
\left.\mathcal{V}_{i}(x, \mathbf{t})\right|_{\mathbf{m}=\left(\mathbf{m}_{i}, \mathbf{m}_{-i}^{*}\right)}=\mathbf{U}_{i}(0)-\sum_{k=0}^{\left|\mathcal{R}_{i}\right|} t_{i}^{l_{i_{k}}}\left(\mathbf{x}_{-i}^{*}, 0, \mathbf{p}_{-i}^{* l_{i_{k}}}, p_{i}^{l_{i_{k}}}\right)=\mathbf{U}_{i}(0)=0 .
$$

when $i \neq k_{l_{1}}, k_{l_{2}}, \cdots, k_{l_{r}}$.

When $i=k_{l_{j}}, j=1,2, \cdots, r$,

$$
\left.\mathcal{V}_{i}(x, \mathbf{t})\right|_{\mathbf{m}=\left(\mathbf{m}_{i}, \mathbf{m}_{-i}^{*}\right)}=\mathbf{U}_{i}(0)-\sum_{k=0}^{\left|\mathcal{R}_{i}\right|} t_{i}^{l_{i_{k}}}\left(\mathbf{x}_{-i}^{*}, 0, \mathbf{p}_{-i}^{* l_{i_{k}}}, p_{i}^{l_{i_{k}}}\right)-\mathcal{Q}^{* l_{j}}=-\mathcal{Q}^{* l_{j}} \geq 0,
$$

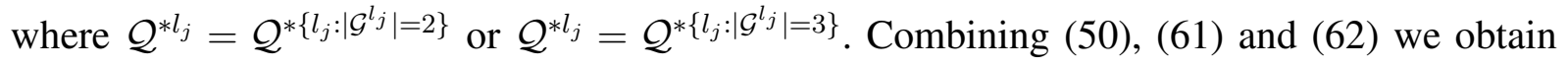

$$
\mathcal{V}_{i}\left(x_{i}^{*}, \mathbf{t}^{*}\right) \geq\left.\mathcal{V}_{i}(x, \mathbf{t})\right|_{\mathbf{m}=\left(\mathbf{m}_{i}, \mathbf{m}_{-i}^{*}\right)} \geq 0
$$


and this establishes (51) and completes the proof.

Proof of Theorem 5. Let $\left(\mathbf{x}^{*}, \mathbf{p}^{*}\right)$ be an arbitrary NE of the game $(\mathcal{M}, f, \mathcal{V})$ induced by the proposed game form. Then by the properties of NE, we must have that for every user $i \in \mathcal{N}$,

$$
\left.\frac{\partial \mathcal{V}_{i}(\mathbf{m})}{\partial x_{i}}\right|_{\mathbf{m}=\mathbf{m}^{*}}=\left.\left[\frac{\partial \mathbf{U}_{i}\left(x_{i}\right)}{\partial x_{i}}-\frac{\partial t_{i}(\mathbf{m})}{\partial x_{i}}\right]\right|_{\mathbf{m}=\mathbf{m}^{*}}=0 .
$$

By Lemma 2, Eq. (64) is equivalent to

$$
\frac{\partial \mathbf{U}_{i}\left(x_{i}\right)}{\partial x_{i}}-\sum_{l \in \mathcal{R}_{i}} p^{* l}=0 .
$$

Furthermore, by Lemma 2 we have $p^{* l} \mathcal{E}^{* l} / \gamma=0$ and since $\gamma>0$

$$
p^{* l} \mathcal{E}^{* l}=p^{* l}\left[\sum_{k \in \mathcal{G}^{l}} x_{k}^{*}-\mathbf{c}^{l}\right]=0
$$

Equation (64) holds for every user $i \in \mathcal{N}$; equation (66) holds for every link $l \in \mathbf{L}$.

Consider now the centralized problem Max. Since the functions $\mathbf{U}_{i}, i \in \mathcal{N}$ are concave and differentiable and the constraints are linear, Slater's condition ( [47]) is satisfied, the duality gap is equal to zero, and the Karush Kuhn Tucker (KKT) conditions are necessary and sufficient to guarantee the optimality of any allocation $\mathbf{x}:=\left(x_{1}, x_{2}, \cdots, x_{N}\right)$ that satisfies them. Let $\lambda^{l}$ be the Lagrange multiplier corresponding to the capacity constraint for link $l$ and $\nu_{i}$ be the Lagrange multiplier corresponding to the demand constraint. The Lagrangian for problem Max is

$$
\mathcal{L}(x, \lambda, \nu)=\sum_{i \in \mathcal{N}} \mathbf{U}_{i}\left(x_{i}\right)-\sum_{l \in \mathbf{L}} \lambda^{l}\left(\sum_{i \in \mathcal{G}_{l}} x_{i}-\mathbf{c}^{l}\right)+\sum_{i \in \mathcal{N}} \nu_{i} x_{i}
$$

and the KKT conditions are:

$$
\begin{aligned}
& \frac{\partial \mathcal{L}\left(x^{*}, \lambda^{*}, \nu^{*}\right)}{\partial x_{i}}=\frac{\partial \mathbf{U}_{i}\left(x_{i}^{*}\right)}{\partial x_{i}}-\sum_{l \in \mathcal{R}_{i}} \lambda^{* l}+\nu_{i}^{*}=0 \\
& \lambda^{* l}\left(\sum_{i \in \mathcal{G}^{l}} x_{i}^{*}-\mathbf{c}^{l}\right)=0 \quad \forall l \in \mathbf{L} \\
& \nu_{i}^{*} x_{i}^{*}=0 \quad \forall i \in \mathcal{N}
\end{aligned}
$$

Since the KKT conditions are necessary and sufficient to guarantee the optimality of any allocation $\mathbf{x}=\left(x_{1}, x_{2}, \cdots, x_{N}\right)$ that satisfies them, it is enough to find $\nu_{i}^{*}$ and $\lambda^{* l}, l \in \mathbf{L}$, such 
that Eqs. 68), 699) and (70) are satisfied.

Set $\nu_{i}^{*}=0, i \in \mathcal{N}$, and $\lambda^{* l}=p^{* l}, l \in \mathbf{L}$. Then (70) is satisfied and (68) and 69) become,

$$
\begin{aligned}
& \frac{\partial \mathbf{U}_{i}\left(x_{i}^{*}\right)}{\partial x_{i}}-\sum_{l \in \mathcal{R}_{i}} p^{* l}=0 \\
& p^{* l}\left(\sum_{i \in \mathcal{G}^{l}} x_{i}-\mathbf{c}^{l}\right)=0 \quad \forall l \in \mathbf{L}
\end{aligned}
$$

respectively, and they are satisfied because they are identical to Eqs. (64) and (66), respectively. Furthermore, by the construction of the game form $\sum_{i=1}^{N} t_{i}^{*}=0$. Consequently, the solution $\mathrm{x}^{*}=\left(x_{1}^{*}, x_{2}^{*}, \cdots, x_{N}^{*}\right)$ of (71) and (72) along with the specification of $t_{i}^{*}, i=1,2, \cdots, N$, are

an optimal solution of Problem Max. At the same time (71) and (72) and $\sum_{i=1}^{N} t_{i}^{*}=0$ are satisfied by the allocation $f\left(\mathbf{m}^{*}\right)$ corresponding to the $\mathrm{NE} \mathbf{m}^{*}$. Consequently, the NE $\mathbf{m}^{*}$ results in allocation $f\left(\mathbf{m}^{*}\right)=\left(x_{1}^{*}, x_{2}^{*}, \cdots, x_{N}^{*}, t_{1}^{*}, t_{2}^{*}, \cdots, t_{N}^{*}\right)$ that is an optimal solution of Problem Max. Since the NE $\mathbf{m}^{*}$ was arbitrarily chosen, every NE $\mathbf{m}^{*}$ of the game form proposed in section [III results in an optimal solution of Problem Max.

\section{Proof of Theorem 6 .}

First we note that an optimal solution $\left(\mathrm{x}^{*}, t^{*}\right)=\left(x_{1}^{*}, x_{2}^{*}, \cdots, x_{N}^{*}, t_{1}^{*}, t_{2}^{*}, \cdots, t_{N}^{*}\right)\left(\right.$ where $t_{i}, i=$ $1,2, \cdots, N$, are defined by (15), (16), (30), (31) and (32)) of Problem Max exists. This follows from: (i) the fact that each $\mathbf{U}_{i}, i \in \mathcal{N}$, is concave and the space of the constraints described by Eqs. (3) and (4) is convex; (ii) the fact that $\sum_{i=1}^{N} t_{i}^{*}=0$ by the construction of the game form. The KKT conditions for problem Max result in the following equations,

$$
\begin{array}{rlrl}
\frac{\partial \mathbf{U}_{i}\left(x_{i}^{*}\right)}{\partial x_{i}^{*}}-\sum_{l \in \mathcal{R}_{i}} \lambda^{* l}+\nu_{i}^{*}=0 & & (N \text { equations }) \\
\lambda^{* l}\left(\sum_{i \in \mathcal{G}^{l}} x_{i}^{*}-\mathbf{c}^{l}\right) & =0 & & (L \text { equations }) \\
\nu_{i}^{*} x_{i}^{*}=0 & & (N \text { equations })
\end{array}
$$

We have $N+L+N$ equations in $L+N$ unknowns, $\lambda^{* l}, l \in \mathbf{L}$ and $\nu_{i}^{*}, i \in \mathcal{N}$. In general we have multiple solutions.

We want to show that for every solution $\left(\lambda^{* l}, \nu_{i}^{*}, l=1,2, \cdots, L, i=1,2, \cdots, N\right)$ of Eqs. (73), (74) and (75) the message $\overline{\mathbf{m}}=\left(\overline{\mathbf{m}}_{1}, \overline{\mathbf{m}}_{2}, \cdots, \overline{\mathbf{m}}_{N}\right), \overline{\mathbf{m}}_{i}=\left(\bar{x}_{i}, \bar{p}_{i}^{l}: l \in \mathcal{R}_{i}\right)$ with $\bar{x}_{i}=x_{i}^{*}$ and 
$\bar{p}_{i}^{l}=\lambda^{* l}$ for all $i \in \mathcal{N}$ and $l \in \mathcal{R}_{i}$, is a Nash equilibrium of the game induced by the proposed game form.

For that matter we note that by the selection of $\overline{\mathbf{m}}$ we have

$$
p_{i}^{* l}=p_{j}^{* l}=\lambda^{* l}=p^{* l}
$$

for every $i$ and $j \in \mathcal{G}^{l}$. By (74) and (76)

$$
p^{* l}\left(\sum_{j \in \mathcal{G}^{l}} x_{j}^{*}-\mathbf{c}^{l}\right)=\lambda^{* l}\left(\sum_{j \in \mathcal{G}^{l}} x_{j}^{*}-\mathbf{c}^{l}\right)=0
$$

and by (30) we obtain

$$
\frac{\partial t_{i}^{* l}}{\partial x_{i}^{*}}=p^{* l}=\lambda^{* l}
$$

for every $l \in \mathcal{R}_{i}$ and every $i \in \mathcal{N}$. Therefore, the message $\overline{\mathbf{m}}$ satisfies all the conditions of Lemma 2 ,

Next we show that for every $i \in \mathcal{N}, \overline{\mathbf{m}}_{i}$ is a solution of the problem,

$$
\begin{aligned}
& \max _{\mathbf{m}_{i} \in \mathcal{M}_{i}}\left\{\mathbf{U}_{i}\left(x_{i}\right)-\sum_{l \in \mathcal{R}_{i}} t_{i}^{l}\left(\overline{\mathbf{m}}_{-i}, \mathbf{m}_{i}\right)\right\} \\
& \text { subject to } \\
& \quad x_{i} \geq 0, p_{i}^{l} \geq 0 \quad \forall l \in \mathcal{R}_{i} .
\end{aligned}
$$

Any maximizing solution of 79 must satisfy

$$
\begin{aligned}
& \frac{\partial \mathbf{U}_{i}\left(x_{i}\right)}{\partial x_{i}}-\sum_{l \in \mathcal{R}_{i}} \frac{\partial t_{i}^{l}\left(\overline{\mathbf{m}}_{-i}, \mathbf{m}_{i}\right)}{\partial x_{i}}+r_{i}=0 \\
& \frac{\partial \mathbf{U}_{i}\left(x_{i}\right)}{\partial p_{i}^{l}}-\sum_{l \in \mathcal{R}_{i}} \frac{\partial t_{i}^{l}\left(\overline{\mathbf{m}}_{-i}, \mathbf{m}_{i}\right)}{\partial p_{i}^{l}}+q_{i}^{l}=0
\end{aligned}
$$

$\forall l \in \mathcal{R}_{i}$, where $r_{i}$ and $q_{i}^{l}$ are the Lagrange multipliers associated with the constraints $x_{i} \geq 0$, and $p_{i}^{l} \geq 0, l \in \mathcal{R}_{i}$, respectively. We set $r_{i}=\nu_{i}^{*}$ and $q_{i}^{l}=0$ for every $l \in \mathcal{R}_{i}$. At $\mathbf{m}_{i}=\overline{\mathbf{m}}_{i}$, Eq. (80) is satisfied because of Eq. (73) and Eq. (78). 
Furthermore at $\mathbf{m}_{i}=\overline{\mathbf{m}}_{i}$ Eq. 81 is satisfied since

$$
\left.\frac{\partial \mathcal{V}_{i}}{\partial p_{i}^{l}}\right|_{\mathbf{m}=\overline{\mathbf{m}}}=-\left.\sum_{l \in \mathcal{R}_{i}} \frac{\partial t_{i}^{l}}{\partial p_{i}^{l}}\right|_{\mathbf{m}=\overline{\mathbf{m}}}
$$

and

$$
\left.\frac{\partial t_{i}^{l}}{\partial p_{i}^{l}}\right|_{\mathbf{m}=\overline{\mathbf{m}}}= \begin{cases}0 & \text { if }\left|\mathcal{G}^{l}\right|=2 \\ -2 p^{* l}\left[\frac{x_{i}^{*}+x_{j}^{*}+x_{k}^{*}-\mathbf{c}^{l}}{\gamma}\right]=0 & \text { if }\left|\mathcal{G}^{l}\right|=3 \\ -2 P_{-i}^{* l}\left[\frac{\mathcal{E}_{-i}^{* l}+x_{i}^{*}}{\gamma}\right]=0 & \text { if }\left|\mathcal{G}^{l}\right|>3\end{cases}
$$

for any $l \in \mathbf{L}$ because of (77). Hence, $\left(x_{1}^{*}, x_{2}^{*}, \cdots, x_{N}^{*}, \lambda^{* l_{1}}, \lambda^{* l_{2}}, \cdots, \lambda^{* l_{L}}\right)$ is a NE point of the game induced by the game form proposed in section III.

Proof of Theorem 7. Since any NE of the game induced by the mechanism proposed in section III, (if such an equilibrium exists), results in a feasible allocation of Problem Max, (see Theorem 1), we restrict attention to the space $\mathcal{M}^{\prime}=\mathcal{M}_{1}^{\prime} \times \mathcal{M}_{2}^{\prime} \cdots \mathcal{M}^{\prime}{ }_{N}$ of strategies that result in feasible allocations of Problem Max. Then, the users' utilities $\mathcal{V}_{i}\left(x_{i}, \mathbf{t}_{i}\right)=\mathbf{U}_{i}\left(x_{i}\right)-\mathbf{t}_{i}$, $i=1,2, \cdots, N$, (where $\mathbf{t}_{i}$ is specified by the game form of section III) are quasi-concave in $\mathbf{m}_{i}=\left(x_{i}, \mathbf{p}_{i}\right)$ and continuous in $\mathbf{m}=\left(\mathbf{m}_{1}, \cdots, \mathbf{m}_{N}\right)=\left(\left(x_{1}, \mathbf{p}_{1}\right),\left(x_{2}, \mathbf{p}_{2}\right), \cdots,\left(x_{N}, \mathbf{p}_{N}\right)\right)$. Furthermore, the message/strategy spaces $\mathcal{M}_{i}^{\prime}$ are compact, convex and non-empty. Therefore, by Glicksberg's theorem [22], there exists a pure $\mathrm{NE}$ of the game $\left(\mathcal{M}, f, \mathcal{V}_{i}, i=1,2, \cdots, N\right)$ induced by the game form of section III.

Let $\mathbf{m}^{*}$ be a NE of this game. Then, for every user $i \in \mathcal{N}$,

$$
\mathcal{V}_{i}\left(\mathbf{m}^{*}\right) \geq \mathcal{V}_{i}\left(\mathbf{m}_{-i}^{*}, \mathbf{m}_{i}\right) \quad \text { for every } \mathbf{m}_{i} \in \mathcal{M}_{i}
$$

That is,

$$
\mathbf{U}_{i}\left(x_{i}^{*}\right)-\sum_{l \in \mathcal{R}_{i}} t_{i}^{* l}\left(\mathbf{m}^{*}\right) \geq \mathbf{U}_{i}\left(x_{i}\right)-\sum_{l \in \mathcal{R}_{i}} t_{i}^{l}\left(\mathbf{m}_{-i}^{*}, \mathbf{m}_{i}\right) \quad \forall \mathbf{m}_{i} \in \mathcal{M}_{i}
$$

where

$$
\sum_{l \in \mathcal{R}_{i}} t_{i}^{* l}\left(\mathbf{m}^{*}\right)=\sum_{l \in \mathcal{R}_{i}} p^{* l} x_{i}^{*}+\sum_{\substack{l \in \mathcal{R}_{i} \\\left|\mathcal{G}^{l}\right|=3}} \Omega_{i}^{* l}+\sum_{\substack{l \in \mathcal{R}_{i} \\\left|\mathcal{G}^{l}\right|>3}} \Phi_{i}^{* l}+\sum_{j=1}^{r} \mathcal{Q}^{* l_{j}} 1\left\{i=k_{l_{j}}\right\}
$$


$\mathcal{Q}^{* l_{j}}$ is given by $\mathcal{Q}^{*\left\{l:\left|\mathcal{G}^{l}\right|=2\right\}}$ or $\mathcal{Q}^{*\left\{l:\left|\mathcal{G}^{l}\right|=3\right\}}$ (cf. Eqs. (15) and (16)), and

$$
\begin{aligned}
\sum_{l \in \mathcal{R}_{i}} t_{i}^{l}\left(\mathbf{m}_{-i}^{*}, \mathbf{m}_{i}\right)= & \sum_{\substack{l \in \mathcal{R}_{i} \\
\left|\mathcal{G}^{l}\right|=2}} \Pi_{2}\left(\mathbf{m}_{-i}^{*}, \mathbf{m}_{i}\right)+\sum_{\substack{l \in \mathcal{R}_{i} \\
\left|\mathcal{G}^{l}\right|=3}} \Pi_{3}\left(\mathbf{m}_{-i}^{*}, \mathbf{m}_{i}\right)+\sum_{\substack{l \in \mathcal{R}_{i} \\
\left|\mathcal{G}^{l}\right|>3}} \Pi_{>3}\left(\mathbf{m}_{-i}^{*}, \mathbf{m}_{i}\right) \\
& +\sum_{j=1}^{r} \mathcal{Q}^{* l_{j}} 1\left\{i=k_{l_{j}}\right\},
\end{aligned}
$$

where

$$
\begin{gathered}
\Pi_{2}\left(\mathbf{m}_{-i}^{*}, \mathbf{m}_{i}\right):=p^{* l} x_{i}+\frac{\left(p_{i}^{l}-p^{* l}\right)^{2}}{\alpha}-2 p^{* l}\left(p_{i}^{l}-p^{* l}\right)\left(\frac{x_{i}+x_{j}^{*}-\mathbf{c}^{l}}{\gamma}\right), \\
\Pi_{3}\left(\mathbf{m}_{-i}^{*}, \mathbf{m}_{i}\right):=p^{* l} x_{i}+\left(p_{i}^{l}-p^{* l}\right)^{2}+\Omega_{i}^{* l}-2 p^{* l}\left(p_{i}^{l}-p^{* l}\right)\left(\frac{x_{i}+x_{j}^{*}+x_{k}^{*}-\mathbf{c}^{l}}{\gamma}\right), \\
\Pi_{>3}\left(\mathbf{m}_{-i}^{*}, \mathbf{m}_{i}\right):=p^{* l} x_{i}+\left(p_{i}^{l}-p^{* l}\right)^{2}+\Phi_{i}^{* l}-2 p^{* l}\left(p_{i}^{l}-p^{* l}\right)\left(\frac{x_{i}+\mathcal{E}_{-i}^{* l}}{\gamma}\right) .
\end{gathered}
$$

Since (85) holds for every feasible $\left(x_{i}, \mathbf{P}_{i}\right)$, setting $p_{i}^{l}=p^{* l}$ for every $l \in \mathcal{R}_{i}$ we obtain,

$$
\mathcal{V}_{i}\left(x_{i}^{*}, \mathbf{P}^{*}\right)=\mathbf{U}_{i}\left(x_{i}^{*}\right)-\sum_{l \in \mathcal{R}_{i}} p^{* l} x_{i}^{*} \geq \mathcal{V}_{i}\left(x_{i}, \mathbf{P}^{*}\right)=\mathbf{U}_{i}\left(x_{i}\right)-\sum_{l \in \mathcal{R}_{i}} p^{* l} x_{i}
$$

for every feasible $x_{i}$. Therefore, for every $i=1,2, \cdots, N$,

$$
x_{i}^{*}=\arg \max _{x_{i} \in \mathbf{D}_{-i}^{*}}\left\{\mathbf{U}_{i}\left(x_{i}\right)-\sum_{l \in \mathcal{R}_{i}} p^{* l} x_{i}\right\}
$$

where $\mathbf{D}_{-i}^{*}:=\left\{x_{i}: 0 \leq x_{i} \leq \min _{l \in \mathcal{R}_{i}}\left\{\mathbf{c}^{l}-\sum_{\substack{j \in \mathcal{G}^{l} \\ j \neq i}} x_{j}^{*}\right\}\right\}$. Consequently, $\left(\mathbf{x}^{*}, \mathbf{p}^{*}\right)$ is a Walrasian equilibrium, therefore $\left(\mathbf{x}^{*}, \mathbf{t}^{*}\right)$ is Pareto optimal ( [12] Chapter 15). 